\title{
Articulación de la ética y la estrategia para integrar expectativas de los grupos de interés a través del propósito superior
}

\section{Articulation of ethics and strategy to integrate stakeholder expectations through higher purpose}

Gina

Giraldo Hernández

Universidad EAFIT,

Colombia

Recibido: 28 de octubre de 2020.

Aprobado: 1 de diciembre de 2020. 


\section{Resumen}

Con el presente artículo se pretende contribuir a la reflexión sobre las implicaciones que tienen las decisiones y acciones de las empresas en la sociedad para buscar una alternativa que les permita incluir en sus estrategias no solo las aspiraciones de los accionistas, sino también las expectativas de los demás grupos de interés. Para lograrlo, se recurre a los autores que han contribuido a consolidar la estrategia como campo de estudio y a profesores de la Escuela de Valencia. En este texto se reconoce la interdependencia humana y la importancia de que los diferentes actores sociales, entre ellos las empresas, participen desde sus roles en la sociedad para lograr los resultados que esperan y, a la vez, generen un impacto positivo en su entorno. Como conclusión, se propone que para incorporar las expectativas de todos los grupos de interés, las empresas articulen no solo su modelo de negocio con el propósito superior y sus objetivos, sino también la ética con la estrategia.

Palabras clave: ética, estrategia, grupos de interés, propósito, objetivo.

Clasificación JEL: M14, L1, L2.

\section{Abstract}

This article is intended to contribute to the debate on the implications of the decisions and actions of companies in society, to find an alternative that allows them to include in their strategies not only the aspirations of the shareholders, but also the expectations of other stakeholders. To achieve this, we rely on the authors that have contributed to consolidating the strategy as a field of study and on professors from the School of Valencia. This text recognizes human interdependence and the importance of different social actors, including companies, participating according to their roles in society to achieve the results they expect and, at the same time, generating a positive impact on their environment. As a conclusion, it is proposed that, in order to incorporate the expectations of all stakeholders, companies articulate not only their business model with the higher purpose and objectives, but also ethics with strategy.

Key Words: ethics, strategy, stakeholders, purpose, objective.

JEL Classification: M14, L1, L2. 


\section{Introducción}

Este artículo refleja la preocupación por la relación entre racionalidad ética y racionalidad estratégica, la relación entre lo público y lo privado, la combinación entre el interés general y el interés individual, entre la responsabilidad y la rentabilidad, la necesidad de generar rentabilidad económica $y$, al mismo tiempo, prosperidad para la sociedad, incluidos todos los grupos de interés; muestra también, el análisis de cómo decisiones organizacionales y aun individuales pueden producir consecuencias públicas que afectan el contexto interno y externo en el que operan. Con este artículo se pretende contribuir al debate acerca de las acciones de la empresa como actor social y sobre alternativas que le permitan lograr los resultados que se propone y generar un impacto positivo en su entorno. Para ello, se hace una amplia revisión y análisis de la literatura sobre el tema.

La interdependencia humana exige examinar la influencia de las decisiones de individuos, organizaciones e instituciones en los demás, entre estas, las que toman las personas en nombre de las organizaciones. Estas decisiones podrían tomarse tras consultar solo las expectativas de los accionistas, o también incluir las expectativas de todos los interesados, entre ellos los accionistas. Es importante reflexionar sobre las decisiones que se toman en las empresas porque podrían afectar la convivencia en el interior de las organizaciones y fuera de ellas.

Se espera que este artículo contribuya a la reflexión sobre alternativas que propicien la articulación de la ética con la estrategia en la operación de los negocios, para incluir las expectativas de los afectados por esta. Lo anterior podría traer como consecuencia el incremento de la confianza, legitimidad, credibilidad y reputación de las empresas, para contribuir a su sostenibilidad económica en el largo plazo y, a su vez, encontrar formas de incrementar los niveles de bienestar, justicia y seguridad de los consumidores en particular y de todos los ciudadanos en general.

Para iniciar la reflexión, se formula una pregunta: ¿cómo pueden las empresas integrar en sus decisiones las expectativas de todos los grupos de interés que impactan su operación? Responderla exige que las empresas reconozcan que, como miembros de la sociedad, se ven impactadas positiva o negativamente por el contexto en el que operan $y$, a su vez, ejercen impacto en ese contexto. Para que integren en sus decisiones y acciones las expectativas de todos sus grupos de interés, se propone diferenciar y articular las declaraciones estratégicas y, asimismo, articular la estrategia de la empresa con la ética como orientadora de la acción. 
Las declaraciones estratégicas son las que marcan la dirección de la empresa y se convierten en su brújula, aun en momentos de gran incertidumbre. Estas declaraciones, además de fijar la dirección, se convierten en los compromisos públicos que establece, con base en los cuales es evaluada.

En segundo lugar, se seleccionan los autores que sirven como punto de referencia del tema que nos ocupa. Se hace una combinación entre autores clásicos de la estrategia y actuales, priorizando los seleccionados a través del ejercicio docente de la autora durante 18 años en la Universidad EAFIT, en la asignatura Estrategia, cuyo contenido se revisa en forma permanente a través del Grupo de estudio de Estrategia. Asimismo, en relación con la ética, se priorizan los escritos de los profesores del máster y doctorado en Ética y Democracia impartido por las Universidades de Valencia y Jaume I de Castellón, miembros de la Escuela de Valencia, liderado por la profesora Adela Cortina, instituciones educativas en las que se formó la autora del presente texto.

Para garantizar coherencia se propone el análisis de las declaraciones estratégicas, lo cual implica responder varias preguntas en relación con la empresa: ¿qué hace?, ¿por qué lo hace?, ¿para qué lo hace?, ¿cómo lo hace?, ¿quién lo hace?, ¿cómo orienta lo que hace? Las respuestas a estas preguntas ofrecen como resultado los conceptos clave en relación con el negocio, que es necesario diferenciar: misión (definición de negocio), objetivo, propósito, estrategia, grupos de interés, ética. Una vez diferenciados los conceptos, se sugiere articularlos para que se relacionen y refuercen entre sí, sin perder su identidad propia, alrededor de lo que denominamos la «esfera de coherencia estratégica».

La esfera de coherencia estratégica propone articular seis componentes: 1) el propósito, 2) el objetivo, 3) el negocio, 4) los grupos de interés, 5) la estrategia y 6) la ética, para garantizar coherencia en la acción, coherencia estratégica, lo cual garantiza la sostenibilidad de la empresa en el largo plazo, al producir un impacto positivo en la sociedad y, a la vez, alcanzar el objetivo que le dio origen. La fortaleza de la esfera es que mueve sus componentes al unísono, de tal forma que si uno se mueve, afecta a todos los demás. Articular los componentes incluidos facilita no solo garantizar la supervivencia económica de la empresa, sino también integrar las expectativas de todos los grupos de interés.

Finalmente, se establecen conclusiones, como las siguientes: integrar las expectativas de todos los grupos de interés evita que se traicione la confianza depositada por el ciudadano en la empresa, al autorizarla a través del gobierno para operar. La 
coherencia entre las declaraciones estratégicas y la articulación de la ética con la estrategia permite integrar las expectativas de los grupos de interés en las decisiones y acciones de la empresa, convirtiéndolos en aliados. La ética reflexiona sobre la justicia de las costumbres y de las leyes. Articular los componentes de la esfera de coherencia estratégica permite considerar las expectativas de todos los grupos de interés, incluidos los accionistas.

\section{Grupos de interés: del interés individual al interés general}

Hechos recientes, como los presentados como consecuencia de la pandemia por la COVID-19, confirman la gran interdependencia que existe entre los seres humanos. Situaciones cotidianas como viajar a otra ciudad o país sin tener conciencia de su estado de salud, hasta decisiones como la de decretar el confinamiento para evitar la propagación incontrolada del virus, con la finalidad de proteger la salud de la población - lo que a su vez afectó la economía de los pequeños negocios-, demuestran los efectos en cadena que pueden tener las decisiones tomadas por individuos, organizaciones o instituciones.

Las prioridades que establecen los diferentes actores de la sociedad, trátese de Estados, empresas, organizaciones sociales, entre ellas las ONG o los individuos, pueden influir positiva o negativamente en la vida de las demás personas; por ello, es importante reflexionar sobre las consecuencias de las decisiones que toman los individuos (nivel micro), las organizaciones (nivel mezzo) o las instituciones (nivel macro). En el presente trabajo se analizan las implicaciones de las decisiones que se toman en el nivel mezzo, es decir, en el interior de las organizaciones, mismas que, si bien son responsabilidad de individuos, lo hacen en nombre de la organización y pueden afectar una parte o al conjunto de los ciudadanos.

\section{Marco conceptual}

En el proceso de toma de decisiones, con frecuencia las empresas priorizan el cumplimiento de las expectativas de los accionistas, o perspectiva de valor para los accionistas (Wit y Meyer, 2010), en vez de integrar los intereses contrapuestos de todos los interesados (Freeman, 1984) y reconocer la contribución de los diferentes grupos de interés en la generación de riqueza (Camacho, Fernández y Miralles, 
2005), o perspectiva de valor de los grupos de interés (Wit y Meyer, 2010). Considerar las expectativas de los grupos de interés es un enfoque responsable y humanista que incorpora un sentido ético en la economía, combinando los valores de la libertad y la justicia (Conill, 2006). Para garantizar la convivencia y la supervivencia humana, todos los actores sociales, independientemente del ámbito en el que se desempeñen, pueden analizar las implicaciones de las decisiones que toman para todos los demás.

Los grupos de interés se pueden identificar como los interlocutores válidos de las empresas, en la medida en la que tienen intereses legítimos frente a ella y esperan que sean tomados en cuenta (Cortina, 2008). La perspectiva de los grupos de interés refleja las expectativas de la sociedad. Esta perspectiva exige preguntarse «[...] qué se produce, para qué se produce y quién decide lo que se produce [...]» (Cortina, 2004: 126), porque cada vez más las nuevas generaciones y las nuevas tendencias de consumo se orientan a seleccionar productos fabricados por empresas que protejan el medio ambiente («Los jóvenes prefieren...», 2020). Otra manifestación de la perspectiva de los grupos de interés es el índice de sustentabilidad Dow Jones, que integra compañías con alto desempeño en las dimensiones de la triple cuenta de resultados, es decir, impactos económicos, sociales y ambientales, que sirve como punto de referencia a posibles inversionistas para decidir dónde invertir sus excedentes.

Asimismo, Kofi Annan, como secretario general de la Organización de las Naciones Unidas en 1999, propuso un Pacto Global ante el Foro Económico Mundial de Davos con el fin de extender los beneficios de la globalización a todos los seres humanos, al reconocer que no solo el poder político, sino también el económico tenían una gran responsabilidad en este asunto, así como el sector social (Parker, 2020). En la misma dirección, los Objetivos de Desarrollo Sostenible (ODS), gestados en la Conferencia de las Naciones Unidas sobre el Desarrollo Sostenible, celebrada en Río de Janeiro en 2012, tienen el propósito de enfrentar los desafíos ambientales, económicos, políticos del mundo, y que coinciden con el Acuerdo de París, aprobado en la Conferencia sobre el Cambio Climático de 2015, después de hacer una evaluación frente a los avances de los Objetivos de Desarrollo del Milenio. Los ODS invitan a crear un planeta más sostenible, seguro y próspero para la humanidad (ONU, 2015).

Otra declaración que se puede destacar es la expresada por la Business Roundtable, que reúne a 181 presidentes ejecutivos de las más grandes corporaciones de Estados Unidos. Tal declaración, de agosto de 2019, rompe con la política vigente durante más de 20 años de priorizar la maximización de beneficios para el accionista y 
empieza a promover la inclusión por parte de las empresas de las expectativas de los demás interesados («Business Roundtable...», 2019). La política de priorizar las expectativas del accionista tiene una fuerte influencia del economista Milton Friedman (1970), a través de su famoso artículo «La responsabilidad social de los negocios es generar utilidades», publicado en 1970 en The New York Times (Bower, 1997).

La declaración de la Business Roundtable se compromete con aspectos como los siguientes: «[...] entregar servicios o bienes de valor a sus clientes, invertir en los empleados y compensarlos de forma justa, negociar de forma justa y ética con los proveedores, apoyar a las comunidades en las que están asentadas las empresas, generar rentabilidad de largo plazo para los accionistas». Si bien es una declaración de intenciones, no un plan de acción, generada por la indignación pública que afecta cada vez más la confianza en las grandes corporaciones, es importante reconocer que representa un avance. Este tipo de declaraciones deben dejar de ser propósitos y convertirse en iniciativas concretas que se vean reflejadas en la realidad. El hecho de que se establezca un compromiso público frente a estos temas implica el reconocimiento de la necesidad de un cambio.

Como lo expresa García-Marzá (2004), las empresas son cada vez más conscientes de que sus decisiones tienen una dimensión moral y que, a su vez, estas tienen también un valor económico. Porter y Kramer identifican cuatro razones para integrar de forma más eficaz las consideraciones sociales a la estrategia y a las operaciones cotidianas del negocio: por obligación moral, porque tienen el deber de ser buenas ciudadanas y deben hacer lo correcto, según valores éticos, respetando las personas, las comunidades y el ambiente natural. Para ser sustentables, con énfasis en la tutoría ambiental y comunitaria, de tal forma que logren satisfacer necesidades presentes sin comprometer la posibilidad de que futuras generaciones también puedan hacerlo; porque quieren mejorar o conservar su reputación, porque al mejorar su imagen, mejoran su marca, aumentan la moral de los empleados e incrementan el valor de sus acciones; porque las empresas reconocen que necesitan una licencia para operar, considerando que debe contar con un permiso tácito o implícito de los gobiernos, las comunidades y otros grupos de interés para hacer sus negocios (Porter y Kramer, 2006).

En este mismo sentido, el Foro Económico Mundial celebrado en Davos en enero de 2020 expresó tres principios, que son por lo menos esperanzadores: 1) el propósito de la empresa es colaborar con todos los grupos de interés relacionados con su funcionamiento, no solo con los accionistas; 2 ) una empresa es más que una unidad económica que genera riqueza, también atiende aspiraciones humanas de 
la sociedad en su conjunto, y 3) las empresas, especialmente las de mayor tamaño, deben estar al servicio del futuro (Schwab, 2020).

Tanto las declaraciones citadas, como las directrices de carácter global expedidas por organizaciones públicas y privadas surgen del reconocimiento de la interdependencia humana y comparten como propósito no eliminar el capitalismo, sino todo lo contrario, contribuir a su sostenibilidad en el largo plazo. Esto es posible si los responsables de tomar decisiones son más conscientes de la importancia de ocuparse no solo de generar riqueza para unos, sino también de redistribuir esa riqueza generada, para contribuir con mayores niveles de bienestar y justicia en la sociedad, al reconocer la participación de los diferentes actores sociales y organizacionales en la generación de esa riqueza.

La licencia para operar (Porter y Kramer, 2006) justifica la razón por la cual la sociedad está legitimada para exigir a las empresas rendición de cuentas por sus acciones y decisiones. En los países con democracia representativa el ciudadano delega, a través del voto, la facultad de definir el marco legal, a cargo del Congreso o Parlamento y, de igual manera, se compromete a cumplir las leyes que son expedidas en su nombre. Asimismo, mediante el voto, el ciudadano delega en el gobierno el manejo de lo público, es decir, de lo que es de todos (Held, 2006). Por lo anterior, se interpreta que cuando el gobierno, como delegatario del ciudadano, le otorga licencia o autorización a una empresa para operar, lo hace en su nombre. Ello significa que quien autoriza a una empresa para operar es el ciudadano, a través del gobierno, y es razonable que espere ver retribuida la confianza que depositó en ella.

Lo que la sociedad espera de la empresa es que asuma la responsabilidad de sus decisiones, que se traduce en que rinda cuentas por lo que hace y evite externalizar o trasladar a la sociedad los costos de esas decisiones. En esta cuestión, la bioética hace un aporte muy valioso cuando destaca que es importante esforzarse no solo por no dañar (principio de no maleficencia), sino que también es importante esforzarse por hacer una contribución positiva, con la advertencia de que la bioética se centra en el paciente y en este artículo se hace referencia a la sociedad. No dañar está en el núcleo de varias teorías éticas que lo consideran como uno de los cimientos de cualquier código moral (Casado, 2008: 109). Esforzarse por no dañar supone aplicar el valor de la responsabilidad que, si bien indispensable, no es suficiente para garantizar prosperidad y sana convivencia en la sociedad. Para lograrlo, también es necesario actuar orientado por otro valor fundamental que es la justicia, que implica la valoración y respeto de todos los seres humanos. Si se trata no solo de no dañar sino también de favorecer, es necesario, además de evitar un perjuicio (responsabilidad), proporcionar un beneficio (justicia). 


\section{Declaraciones estratégicas - proceso estratégico}

Los compromisos públicos que establecen las empresas con la responsabilidad y la justicia suelen plasmarse en sus declaraciones estratégicas, las que Senge (1992) denomina «ideas rectoras». Estas marcan el rumbo de la empresa, al establecer no solo hacia dónde orienta sus actuaciones sino, además, hasta dónde tiene previsto llegar. Para definir la estrategia, la empresa puede apoyarse en la planeación estratégica como herramienta para tener mayores posibilidades de acertar. Ante entornos cada vez más dinámicos, existe mayor conciencia de que la estrategia planeada no siempre coincide con la ejecutada (Mintzberg, Ahlstrad y Lampel, 2007). Los gerentes, en nombre de los propietarios, pueden tener iniciativas intencionales o emergentes (Hambrick, Nag y Chen, 2007), lo que exige apoyarse en procesos estratégicos que señalen una dirección clara y un diagnóstico actualizado, independientemente de que la estrategia surja de forma emergente o planeada.

El proceso estratégico, como herramienta que concibe el surgimiento de la estrategia de forma algo más dinámica, necesita permanente actualización, ajuste y adaptación, así como consultar las expectativas del mercado y las características de los entornos interno y externo en cada momento. En el proceso estratégico se identifican cuatro etapas principales para la empresa: direccionamiento, que incluye las declaraciones estratégicas e implica definir la visión, es decir, cómo se ve la organización en el largo plazo; la misión expresa a qué se dedica; los objetivos y metas que permiten identificar hasta dónde quiere llegar, es decir, qué pretende alcanzar y cuándo; el propósito, que se refiere a cuál es su finalidad, y los valores, que son los orientadores de la conducta con los que se compromete públicamente.

Otra de las etapas del proceso estratégico es la de diagnóstico. Esta contempla aplicar diversas herramientas que permiten saber cómo se encuentra la organización en relación con su entorno externo: análisis de la industria a través de «cinco fuerzas» para examinar la capacidad de negociación de clientes y proveedores, la rivalidad actual y las amenazas de sustitutos o de ingreso de nuevos competidores (Porter, 1999); análisis de la localización geográfica a través del «diamante competitivo», con variables como condiciones de los factores, de la demanda, de rivalidad y existencia de industrias de apoyo en el lugar en donde se opera (Porter, 1999); análisis de macrotendencias a través de la herramienta conocida como PESTEL, con variables políticas, económicas, sociales, tecnológicas, ecológicas y legales (Johnson, Scholes y Whittington, 2005). 
En relación con el entorno interno, la empresa puede analizar las capacidades que ha logrado desarrollar y cómo las usa (Hamel y Prahalad, 1990), la cadena de valor (Porter, 1999) e identificar los recursos que tiene, administra y domina (Montgomery y Collins, 1995). Con base en la información obtenida con la aplicación de las herramientas mencionadas en relación con los entornos interno y externo, se estaría en condiciones de desarrollar el DOFA de la empresa: es decir, identificar debilidades, oportunidades, fortalezas y amenazas. El diagnóstico cumple un papel muy importante porque «[l]a empresa no es una organización neutra independiente de las personas que la componen y aislada de la sociedad en la que está inmersa» (García-Marzá, 2004: 93). La empresa influye en el entorno y a su vez este la influye.

Otra etapa del proceso estratégico es la selección de la estrategia. Teniendo claros el rumbo de la organización y su diagnóstico, será fácil decidir cuál es la estrategia más adecuada para la empresa. Este conocimiento previo o experiencia, permitiría definirla con anticipación (planeada) y también, ante entornos cambiantes o situaciones inesperadas, desarrollar la acción, es decir, la estrategia (emergente). Es importante que las estrategias seleccionadas en cada nivel (corporativo, competitivo, funcional), se complementen o refuercen entre sí. Sobre este particular, podrían considerarse las recomendaciones de Michael Porter sobre la importancia de garantizar la complementariedad y el refuerzo entre las estrategias competitivas y corporativas elegidas (Porter, 1997a, Porter, 1997b).

Una cuarta etapa es la implementación o ejecución. Esta es fundamental, considerando que la estrategia es ante todo acción y, por tanto, no tiene sentido solo formularla, sino también esforzarse por convertirla en realidad. Para lograrlo, existen varias herramientas, como el «modelo estrella», para adelantar el diseño organizacional con el análisis de variables tales como las personas, la estructura, los procesos, los incentivos, las capacidades y habilidades, todo en relación con la estrategia (Kates y Galbraith, 2007). En este modelo se destaca la estructura de la empresa, la cual establece quiénes llevarán a cabo las acciones.

También es necesario definir un sistema de gestión para hacer el seguimiento a los objetivos propuestos. Aquí, herramientas como el Cuadro de mando integral representan una gran contribución al incluir, además del seguimiento de las expectativas del accionista, las de los clientes y los empleados, así como los procesos internos, y el aprendizaje y desarrollo (Norton y Kaplan, 2000). También es necesario identificar la cultura o comportamiento, que refleja los valores con los que se comprometió la empresa. 
Si entendemos la ética como un instrumento de gestión (García-Marza, 2004: 243) que, articulada con la estrategia, permite integrar en las decisiones y acciones de la empresa las expectativas de todos los afectados, se hace necesario incluir otros componentes del proceso estratégico en la etapa de implementación: los códigos de ética y los comités de ética. Los códigos de ética orientan y regulan la conducta en el ámbito laboral y hacen explícita la necesidad de asumir responsabilidades frente a los grupos de interés, más allá de las obligaciones legales, al reconocerlos como actores importantes. Los códigos de ética incluyen medidas disciplinarias en caso de incumplimiento (Lozano, 2004). Por ser un «[...] documento formal donde se expresa la voluntad y la disposición de la empresa para el reconocimiento y satisfacción de todos los intereses en juego» (García-Marza, 2004: 239), se recomienda integrar a todos los afectados en su elaboración y seguimiento. Los códigos de ética son mecanismos utilizados para institucionalizar la ética en las organizaciones y forman parte del sistema para su gestión.

Los comités de ética son «[...] ámbito para el seguimiento y control de los compromisos adquiridos, así como para la deliberación y la búsqueda de intereses comunes y generalizables» (García-Marzá, 2004: 239). Desde la perspectiva ética se recomienda pasar de la información a la comunicación, de aquí la importancia de contar con espacios para el acuerdo y consenso sobre intereses en juego y, además, de reconocer la capacidad de los interesados de decidir sobre los asuntos que pueden afectarlos. Esto hace que los comités de ética cobren importancia como espacios de deliberación y diálogo sobre intereses generalizables, que atienden las expectativas de todos los que actúan en el contexto empresarial, aspecto que se consigna en los códigos correspondientes. Sus deliberaciones no tienen carácter vinculante, su misión es asesorar, supervisar y proponer (García-Marzá, 2004). La pluralidad de sus integrantes puede ser un factor de éxito en su labor.

Identificados los diversos componentes del proceso estratégico, lo más importante es establecer las relaciones entre estos. Por ejemplo, los objetivos y las acciones estratégicas se introducen en el cuadro de mando integral, para poder hacer un mejor seguimiento; los valores se reflejan en la cultura organizacional, e influyen en la estructura, que a su vez dependerá de la estrategia elegida. Dependiendo del resultado de la aplicación de las diferentes herramientas de diagnóstico utilizadas, se dispondrá de mejor información para decidir conservar la estrategia aplicada hasta el momento, o su modificación.

El proceso estratégico es una especie de mapa que incluye las coordenadas para llegar al sitio que se ha fijado la empresa, y dentro de este, las declaraciones 
estratégicas sirven de brújula o guía, aun cuando el mapa pierda vigencia en momentos de gran incertidumbre. Las empresas podrían asegurarse de que la estrategia, esto es, la acción, permita cumplir los compromisos públicos que han establecido (lo que implica la consulta de las expectativas de la sociedad) y, a la vez, alcanzar los objetivos que se ha propuesto (lo que supone la consulta de las expectativas de los accionistas). Se propone, en primer lugar, responder varias preguntas en relación con la operación de las empresas; después, diferenciar los conceptos que reflejan las respuestas a estas preguntas y finalmente, articular la ética con la estrategia para garantizar coherencia entre las declaraciones estratégicas. Las preguntas que se proponen: ¿Qué hace? ¿Por qué lo hace? ¿Para qué lo hace? ¿Cómo lo hace? ¿Quién lo hace? ¿Cómo orienta lo que hace?

\section{Preguntas fundamentales sobre el negocio: diferenciar los conceptos}

\section{¿Qué hace? Misión-definición del negocio}

La misión responde a la pregunta ¿qué hace la empresa? y se concreta en su definición del negocio. "La misión de una empresa describe lo que esta hace» (Hill y Jones, 2011: 14) Por lo tanto, "[u]n primer paso importante en el proceso de formular una misión es llegar a la definición de negocio de la organización. En esencia, la definición responde a estos interrogantes: “¿Cuál es nuestro negocio? ¿Cuál será? ¿Cuál debe ser?"» (Hill y Jones, 2011: 14). Para definir el negocio, se recomienda que la empresa se apoye en un modelo de negocio. «Un modelo de negocio describe la estructura del producto, servicio y flujos de información, y el papel de los agentes implicados» (Hill y Jones, 2011: 15). «Un modelo de negocio es la concepción de un administrador de cómo deben integrarse las estrategias de su empresa en un todo congruente, lo que le permitirá lograr una ventaja competitiva, y una rentabilidad y crecimiento de utilidades superiores» (Hill y Jones, 2011: 6).

Es un error apoyarse en solo dos dimensiones (productos y mercados) para definir el negocio. Por esta razón, se propone un análisis tridimensional para su definición: 1) grupo de clientes o respuesta a la pregunta ¿a quién se está satisfaciendo? 2) necesidades o funciones que se satisfacen, o respuesta a la pregunta ¿qué se está satisfaciendo?, y 3) la tecnología, habilidades o destrezas, o respuesta a la pregunta ¿cómo se están satisfaciendo las necesidades de los clientes? (Abell, 1980). 
Un modelo de negocio se define en términos de segmentación, productos, distribución, servicio y precio (Hamel y Prahalad, 2005). De esta manera se destacan sus elementos más importantes.

Otra buena herramienta para ello, debido a su carácter integral y la potencia que representa, es poder visualizar en un mismo espacio (un lienzo) las variables que se acordaron como prioritarias para definir el negocio. Este es el modelo "canvas», del que si bien su cara visible es Alexander Osterwalder, fue creado de forma participativa, considerando que 470 personas alrededor del mundo se pusieron de acuerdo acerca de cuáles eran las variables prioritarias a tener en cuenta: segmento de clientes, propuesta de valor, canales, relaciones con los clientes, fuentes de ingreso, recursos clave, actividades clave, asociaciones clave y estructura de costos. Osterwalder y Pigneur (2011: 14) expresan en qué consiste de la siguiente manera: «Un modelo de negocio describe las bases sobre las que una empresa crea, proporciona y capta valor». La fortaleza de este modelo radica en la posibilidad de poner a interactuar de forma permanente las variables acordadas para identificar más fácilmente si la variación en una o algunas de estas variables puede incidir en la necesidad de hacer cambios en las demás.

\section{¿Cómo lo hace? ¿Quién lo hace? Estrategia y estructura}

La respuesta a la pregunta ¿cómo lo hace? tiene relación con la estrategia. La estrategia es «Determinación de las metas básicas de largo plazo y los objetivos de una empresa, la adopción de cursos de acción y la asignación de recursos necesarios para llevar a cabo esas metas» (Chandler,1962). Es ante todo acción, pero no aquella individualmente considerada ni una colección de actividades sino, como lo expresa Porter, un sistema de actividades que encajan y se refuerzan entre sí para generar una ventaja competitiva sostenible en el largo plazo (Porter, 1997a).

La estrategia es el patrón de los principales objetivos, propósitos o metas y las políticas y planes necesarios para lograrlas (Andrews, 1985). Estas acciones implican la toma de decisiones y el desarrollo de acciones por parte de personas que actúan a nombre de la empresa, lo que exige responder también a la pregunta ¿quién lo hace? Y para responder, se define cuál es la estructura organizacional que se utilizará, aunque es preciso no limitarse a los empleados, porque en la obtención de los resultados del negocio no solo influyen estos, sino los demás actores, es decir, todos los grupos de interés de la empresa. 
Alfred Chandler (1962) define la estructura como el diseño a través del cual se administra la organización, ya sea que se defina formal o informalmente; también considera que incluye dos aspectos: las líneas de autoridad y comunicación y los datos e información que fluyen a través de esas líneas de comunicación y autoridad. En referencia al diseño estructural Johnson, Scholes y Whittington (2005) expresan que este describe papeles, responsabilidades y relaciones jerárquicas en las organizaciones, que pueden influir profundamente en la ventaja competitiva, en especial en lo que se refiere a la gestión del conocimiento que facilita la aplicación de la estrategia. Estos autores advierten que para que tenga éxito, además de la estructura son también necesarios los procesos que guían al individuo dentro y fuera de la organización, así como las relaciones entre esos individuos. Más adelante se verá que las personas encargadas de la toma de decisiones necesitan apoyarse en orientadores de la conducta, para poder garantizar la sostenibilidad de los resultados en el largo plazo.

\section{¿Por qué lo hace? Visión-objetivos}

Para Chandler (1962) la estrategia no solo es un conjunto de acciones que requieren el uso de recursos sino que, además, están orientadas a lograr metas de largo plazo y los objetivos de la empresa. Esta precisión lleva a responder otra de las preguntas: ¿por qué lo hace?, es decir, ¿por qué las empresas Ilevan a cabo esas acciones? Es importante reconocer que esta pregunta, ¿por qué?, tiende a confundirse con frecuencia, en el lenguaje común, con ¿para qué? El porqué es la razón que origina un esfuerzo. En el caso de las empresas implica preguntarse ¿por qué un grupo de inversionistas decide invertir ciertos recursos en un negocio? Los accionistas esperan un retorno económico, deben tener un incentivo económico para correr un riesgo, de lo contrario podrían buscar otras alternativas de inversión. Interpretan que es una rentabilidad que buscan no solo como resultado sino, además, como una fuente de poder competitivo (Wit y Meyer, 2010). «Actuar racionalmente es actuar a partir de razones, saber por qué hacemos algo o dejamos de hacerlo» (García-Marzá, 2004: 97).

Las empresas se ocupan de esta expectativa definiendo la visión, que tiene un carácter motivado, retador, ambicioso, y la concretan en metas y objetivos, que son realistas y medibles. La visión, como declaración estratégica, expresa cómo se ve la organización en el largo plazo. Para Hill y Jones (2011: 15), «[...] la visión de una empresa presenta parte del estado futuro deseado, articula, con frecuencia en términos audaces, lo que a la empresa le gustaría lograr». Para Mintzberg, Quinn y Voyer (1997), una visión es una imagen mental de cómo quieren los líderes que sea 
su negocio, y asocia el concepto más con el liderazgo personal (Mintzberg, Quinn y Voyer, 1997: 42). «Se trata la aspiración en torno al estratega, tal vez el CEO intenta centrar la atención y las energías de los miembros de la organización» (Johnson, Scholes y Whittington, 2005: 14). La visión es la imagen de futuro que se procura crear (Senge, 1992).

Pero en materia de administración de recursos, las aspiraciones son importantes porque propician la motivación, pero no son suficientes, se requiere de manera prioritaria definir de forma concreta qué es lo que se quiere lograr, en qué cantidad o proporción y cuándo se logrará. Lo anterior exige la definición de objetivos. «Las metas (u objetivos) establecen qué es lo que se va a lograr y cuándo serán alcanzados los resultados, pero no establecen cómo serán logrados» (Mintzberg, Quinn y Voyer, 1997: 7). «Los objetivos son declaraciones de resultados concretos que se quieren conseguir» (Johnson, Scholes y Whittington, 2005: 210). «Una meta es un estado futuro deseado, preciso y medible que una empresa intenta alcanzar. En este contexto, el fin de las metas es especificar con precisión qué se debe hacer si la empresa logra su misión y su visión» (Hill y Jones, 2011: 16).

Lo anterior cobra mayor importancia si se reconoce que con frecuencia los responsables de las empresas no están administrando recursos propios, sino de terceros que depositaron su confianza en ellos, es decir, se aplica la teoría de agencia, que implica que los accionistas o dueños de la empresa no manejan de forma directa sus intereses, sino que delegan este manejo a terceros a quienes pagan para que lo hagan. Aquí, para efectos de rendir cuentas, se requiere la definición de objetivos concretos y medibles que permitan darles seguimiento y saber si se está logrando el resultado económico que se espera o no. Esto significa que los objetivos y la visión, aunque se espera sean una fuente de motivación para todos los empleados, reflejan de manera fundamental las expectativas de los accionistas. Se recomienda el cuadro de mando integral para evaluar los objetivos, porque incluye el seguimiento no solo de las expectativas de los accionistas, sino también de los clientes y los empleados (Norton y Kaplan, 2000).

\section{¿Para qué lo hace? Propósito superior}

Así como el qué se plasma en el modelo de negocio, el cómo se plasma en la estrategia y el por qué en los objetivos, el quién se plasma en la estructura y el para qué lo hace en el propósito. Hamel y Prahalad (2005) se refieren al propósito estratégico como aquel que imprime una obsesión por ganar en todos los niveles de 
la organización, permite imaginar una posición deseada de liderazgo y establece el criterio que la organización tendrá para trazar su progreso. Lo consideran más que una simple ambición desbocada, un activo proceso de gestión, que motiva a la gente comunicando el valor del objetivo, guía la asignación de recursos y le permite a la organización enfocarse (Hamel y Prahalad, 2005). Interpretamos que cuando Adela Cortina (2003) destaca la importancia de definir el bien interno de cada actividad y la necesidad de averiguar qué valores, virtudes y normas lo posibilitan, hace referencia al propósito o finalidad de las empresas.

«El fin de las organizaciones es sin duda un fin social, porque toda organización se crea para proporcionar a la sociedad unos bienes, en virtud de los cuales queda legitimada su existencia ante la sociedad [...]» (Cortina, 2003: 22). Para Adela Cortina, cada actividad, entre ellas la empresarial «[...] produce unos bienes de los que cobra todo su sentido y que son los que la sociedad le reclama, porque existen precisamente para proporcionarlos. Y, como es obvio, también para alcanzarlos unos medios resultan adecuados y otros totalmente inapropiados». Se refiere a los bienes internos, que expresan cuál es el fin específico, el que le permite legitimidad social. Los diferencia de los bienes externos, que no identifican a las organizaciones sino que son comunes a ellas, como el prestigio, el dinero o el poder (Cortina, 2003: 23-24). «Ya no cabe una concepción de empresa estructurada solo en torno al dinero, el poder y la coacción jurídica» (García-Marzá, 2004: 22). El profesor García-Marzá se refiere a que la empresa se puede estructurar también en torno a recursos morales como la confianza.

Para Beatriz Restrepo el propósito o fin de las organizaciones se configura como el soporte fundamental de la vida moral, y hace énfasis en que los fines son siempre sociales (Restrepo, 2014), se ocupan de las expectativas del conjunto de la sociedad, es decir, de todos los grupos de interés de la empresa. Para Cynthia Montgomery (2013: 69), el propósito permite crear una diferencia que importa. «El propósito es la manera en la que [...] una empresa se describe a sí misma, en los términos más esenciales posibles: su razón de existir, el valor único que aporta al mundo, lo que la distingue y le da importancia». Esta autora hace énfasis en que lo más importante para la supervivencia y el éxito de una empresa es su razón de existir, y las necesidades que intenta satisfacer, porque el propósito hace que sus actividades sean más nobles y dignas. También advierte que el propósito, al igual que la estrategia, exige renunciar, porque es tan importante elegir aquello que se hará y lo que se descartará. Precisa que definir el propósito es responsabilidad del líder, del estratega y que «crear valor para los demás es la forma más segura de captar algo de ese valor para ti» (Montgomery, 2013: 73-75, 82-83). 
Para Charles (1965) el propósito es un fenómeno que proporciona orden, dirección y coherencia, y es importante preguntarse por la relación entre este y otras variables organizacionales. Recomienda definir un propósito organizacional único, porque considera que hacerlo para cada grupo de interés genera inconsistencias. Cree que debe definirse en términos de las consecuencias y funciones más frecuentes de una organización, porque se reconoce que cualquier actividad puede tener multiplicidad de consecuencias. Recomienda tener en cuenta las lógicas y creencias de la organización que influyen en sus actividades y opina que cada una tiene un conjunto específico de lógicas que los miembros aceptan como marco de referencia para participar en ella. Esas lógicas frecuentemente están implícitas e implican supuestos y creencias.

Para Hauge y Knudsen (2017) el propósito debe no solo articularse, sino también activarse a través del sistema de control de la gestión, de la cultura corporativa, de los procesos de planificación y de las políticas internas. Así, es preciso evaluar no solo las implicaciones financieras (ganancias) sino, además, su alineación con los valores. El propósito permite mayor lealtad de los diversos grupos de interés, como clientes, socios comerciales, empleados más comprometidos, porque le dan sentido a su trabajo y comprenden mejor la contribución que hacen a su organización; son más rentables, con mayor grado de innovación, y lo relacionan con elementos «blandos» de esta, como la cultura y los valores de la corporación. Garantiza coherencia entre lo que los directivos dicen que hacen y lo que efectivamente ejecutan. Identifican que cada vez más publicaciones como Harvard Business Review o MIT Sloan Management Review se ocupan del propósito de las empresas y que incluso en el Foro Económico Mundial, que se reúne anualmente en Davos, los líderes empresariales y políticos de todo el mundo conversan sobre cómo las empresas pueden combinar con éxito ganancias y propósito.

Sinek (2009) propone un marco conceptual conocido como Círculo dorado, que consta de tres elementos: el porqué, el cómo y el qué de una organización, es decir, propone preguntarse no solo qué hacen las organizaciones exitosas y cómo lo hacen, sino también por qué lo hacen. Considera que los grandes líderes inspiran la acción porque generan confianza y lealtad de sus empleados y clientes al centrarse en el propósito de su organización, y no solo en los productos o servicios que ofrecen, porque cree que las personas no compran lo que se hace, sino la razón por la que se hace, el porqué. El propósito puede tener un papel importante en la relación de una organización con sus grupos de interés. Es pertinente lo que expresa Sinek (2009), pero es necesario advertir que este responde al para qué y no al por qué, tal y como 
se ha expresado en el presente artículo, porque las actividades de las empresas deben estar orientadas no solo por sus objetivos, sino también por su propósito.

Es preciso reconocer que el criterio que determina la compra para un cliente no es el nivel de rentabilidad que espera obtener la empresa, sino la expectativa de valor que le proporcionará, y este valor para el cliente y demás grupos de interés se plasma en el propósito, no en el objetivo. Por esto, los grupos de interés establecen una conexión más fuerte, un vínculo mayor con el propósito. Considerando que este refleja las expectativas de los diferentes grupos de interés, entre ellos las de los clientes, es muy importante contar con una herramienta que permita, de forma efectiva, dar seguimiento al cumplimiento de esas expectativas. Para ello, es pertinente apoyarse en el Mapa de grupos de interés, el cual se espera incluya las expectativas y aportes de todos los grupos de interés, entre ellos los accionistas. Para las empresas este paso implica pasar del gobierno corporativo, que prioriza los intereses de los accionistas, a la gobernanza corporativa, que exige reconocer la influencia que tienen otros grupos en los resultados de la empresa.

Para construir este mapa se propone no solo incluir la expectativa que tiene cada grupo de interés, sino, además, reconocer el aporte de cada uno a la generación de riqueza. Reconocerlo y valorarlo implica considerarlo un socio de la empresa, un aliado. En este caso los grupos de interés se diferencian por el carácter de sus aportes. El reconocimiento podría influir visiblemente en la calidad de las relaciones, una relación de igualdad no de subordinación, para consolidar un recurso relacional, que como recurso intangible se multiplica, no se agota con su uso, si se usa bien y permite tener aliados en todo momento.

Wit y Meyer (2010) resaltan la importancia del propósito al recordar que la estrategia es un curso de acción para lograr un propósito. Se quejan por la poca atención que las organizaciones prestan a su propósito, al centrarse más en los medios que en los resultados, lo que se ve reflejado en la literatura sobre dirección. Resaltan la importancia de principios fundamentales que den sentido a las decisiones estratégicas y destacan como elemento central para lograrlo la misión corporativa. Incluyen el propósito como parte de la misión, reconocen que el propósito de una organización se puede definir como la razón por la cual existe, pero lo integran con las creencias organizacionales, los valores organizacionales y la definición del negocio, como parte de la misión corporativa.

En su texto «Dirección estratégica» no definen el concepto de propósito, aunque reconocen la necesidad de una declaración que la mayoría o todas las partes 
interesadas puedan suscribir. Sí reflexionan sobre los factores que influyen sobre los propósitos de una organización, como son el marco de gobierno corporativo (relaciones con los accionistas), las relaciones con las partes interesadas, los estándares éticos y la cultura, que tienen relación con los valores, los cuales definen la forma en que funciona la organización, los principios que guían su acción. Los valores suelen ser una expresión de la forma en la que una organización se comporta, mientras otros son los que aspira alcanzar, pero estos autores advierten sobre la importancia de que las acciones en la práctica coincidan con la declaración pública de los valores empresariales, que son los que definen los límites en los que se desarrolla cada una de las estrategias (Johnson, Scholes y Whittington, 2006: 208-210).

El propósito como razón de ser de la empresa, que refleja su finalidad y el beneficio que quiere proporcionar al mundo, es la declaración estratégica (ver figura 1), que refleja mejor las aspiraciones de la sociedad, es decir, de todos los grupos de interés. Por lo anterior y para garantizar coherencia en la actuación empresarial, la búsqueda de la maximización de la rentabilidad para el accionista, que es el objetivo de las empresas, puede complementarse con el propósito, que refleja las expectativas de todos los grupos de interés, entre ellos los accionistas, y para lograrlo se propone articular la ética con la estrategia.

\section{Articular la ética con la estrategia}

\section{Preguntarse ¿cómo orienta lo que hace? Orientadores de la conducta}

Diferenciadas las declaraciones estratégicas, que responden a las preguntas planteadas, se propone articular la ética con la estrategia para garantizar coherencia entre las declaraciones estratégicas y así integrar las expectativas de todos los grupos de interés en las decisiones y acciones de la empresa, es decir, en la estrategia. Lo anterior permitirá generar o afianzar la confianza que el conjunto de la sociedad tiene en la empresa, tomando en cuenta la expectativa de comportamiento que enfrenta; los ciudadanos no esperarían que la empresa logre la maximización de beneficio para los accionistas ocasionando un perjuicio a la sociedad. De aquí la importancia de lograr los objetivos sin afectar su propósito.

Para lograr la complementariedad entre el propósito y el objetivo, se propone que las empresas se aseguren de plasmar en el modelo de negocio no solo la maximización 
de la rentabilidad que esperan, que se representa en la base del modelo canvas para definir el negocio, es decir, la diferencia positiva entre los costos clave y los ingresos clave. Además, deberán cerciorarse de que la propuesta de valor, que es el corazón del lienzo del modelo de negocio, se corresponda con el propósito superior con el que se ha comprometido la empresa.

Articuladas las citadas declaraciones estratégicas alrededor del modelo de negocio, se podría proceder a seleccionar la estrategia competitiva más adecuada. Pero para garantizar que se logre la maximización de la rentabilidad para el accionista y el beneficio para la sociedad a la vez, es necesario articular la estrategia, que es la acción, con el orientador de la acción, que es la ética. La sociedad no entendería que la empresa lograra la maximización de la rentabilidad a costa de ocasionar un perjuicio a los clientes y demás afectados.

Para Friedman (1997) las empresas son personas jurídicas y considera que por eso no toman decisiones, sino que lo hacen las personas naturales. Sin embargo, es necesario recordar que las personas toman decisiones en nombre de la organización y apoyadas en las facultades que le otorga su posición en la estructura organizacional. Por eso se presenta una combinación de responsabilidad individual de la persona que toma la decisión, o desarrolla la acción o estrategia, y de responsabilidad institucional de la empresa, a nombre de la que se actúa, lo que puede afectar su reputación, imagen y credibilidad. Esas acciones de los individuos a nombre de las empresas se pueden apoyar en los orientadores de la conducta. Los orientadores de la conducta aseguran corrección en el comportamiento. Entre estos mencionaremos las políticas, es decir, «reglas o guías que expresan los límites dentro de los que debe ocurrir la acción» (Mintzberg, Quinn y Voyer, 1997: 7).

Otros orientadores de la conducta incluyen el derecho, a través de las leyes aprobadas por una autoridad competente, que en los países democráticos son los parlamentos o congresos elegidos por los ciudadanos a través del voto. Estos autorizan al Congreso para definir el marco legal y, a su vez, esperan que esas normas defiendan el interés común y se comprometen a cumplirlas. «El derecho en una sociedad es el conjunto de leyes que han sido promulgadas de forma legítima, según los procedimientos estipulados por ella. Si una norma ha sido promulgada siguiendo los procedimientos legítimos es ya una norma jurídica y su cumplimiento es legalmente exigible por la autoridad competente» (Cortina, 2003: 26). El derecho es muy importante como instrumento del Estado para establecer normas que permiten acciones sociales, entre ellas la economía, y es necesario, pero no es suficiente (García-Marzá, 2004). 
Además del derecho, otros recursos de importancia que se derivan del capital social son la confianza, las redes informales de comunicación o los valores éticos, lo que exige la complementariedad entre ética y derecho (García-Marzá, 2004). Por tanto, es importante considerar también la ética y la moral a través de los valores. «Los valores de una empresa expresan cómo deben comportarse directivos y empleados, cómo deben hacer negocios y qué tipo de organización deben construir para ayudar a la empresa a lograr su misión. Puesto que ayudan a moldear e impulsar el comportamiento dentro de una empresa, los valores suelen considerase como la base de la cultura organizacional de la empresa: el conjunto de valores, normas y estándares que controlan cómo trabajan los empleados para lograr la misión y metas de la organización» (Hill y Jones, 2011: 15-16).

La moral se relaciona con la costumbre propia de una época específica o de un lugar del mundo, influida por creencias filosóficas, ideológicas o religiosas. «La moral no es, pues, un invento de los filósofos, sino un saber que acompaña desde su origen la vida de los hombres, aunque haya ido recibiendo distintos contenidos» (Cortina, 2003: 28). La ética se ocupa de reflexionar sobre la moral (Cortina, 2003), permite hacer reflexiones personales, pero a la luz de valores que tienen vocación de universalidad. «[L]a ética es un tipo de saber de los que pretende orientar la acción humana en un sentido racional; es decir, pretende que obremos racionalmente» (Cortina, 2003: 17). Ello supone analizar y sopesar las posibles consecuencias de lo que se hace, antes de tomar una decisión.

«Una de las principales tareas de la ética es la de pertrechar al ser humano con argumentos sólidos para distinguir lo correcto de lo incorrecto, lo justo de lo injusto» (Siurana, 2009: 14). La ética, como orientadora de la conducta, permite incluir en las decisiones las expectativas de todos los afectados, es decir, de todos los grupos de interés. Es un saber práctico que permite tomar decisiones prudentes y justas (Cortina, 2003). Para Adela Cortina (2009: 119) la responsabilidad como dimensión de la ética empresarial, «[...] debe asumirse como una herramienta de gestión, como una medida de prudencia y como una exigencia de justicia». Una medida de prudencia porque si la empresa se asegura de cumplir las expectativas de todos los grupos de interés, conseguirá aliados, socios, en quienes se puede apoyar en caso de necesitarlos, para lograr lo que se ha propuesto, no enemigos, ni opositores. Y es una medida de justicia, porque si la empresa incluye entre sus decisiones y acciones, es decir, en sus estrategias, las expectativas de los grupos de interés, es porque reconoce no solo que son valiosos en la medida en que cada uno hace aportes relevantes para la generación de riqueza, sino que, además, reconoce que como seres humanos son valiosos en sí mismos, tienen valor, no precio y, por tanto, merecen consideración y respeto. 
Figura 1. Articulación de las declaraciones estratégicas

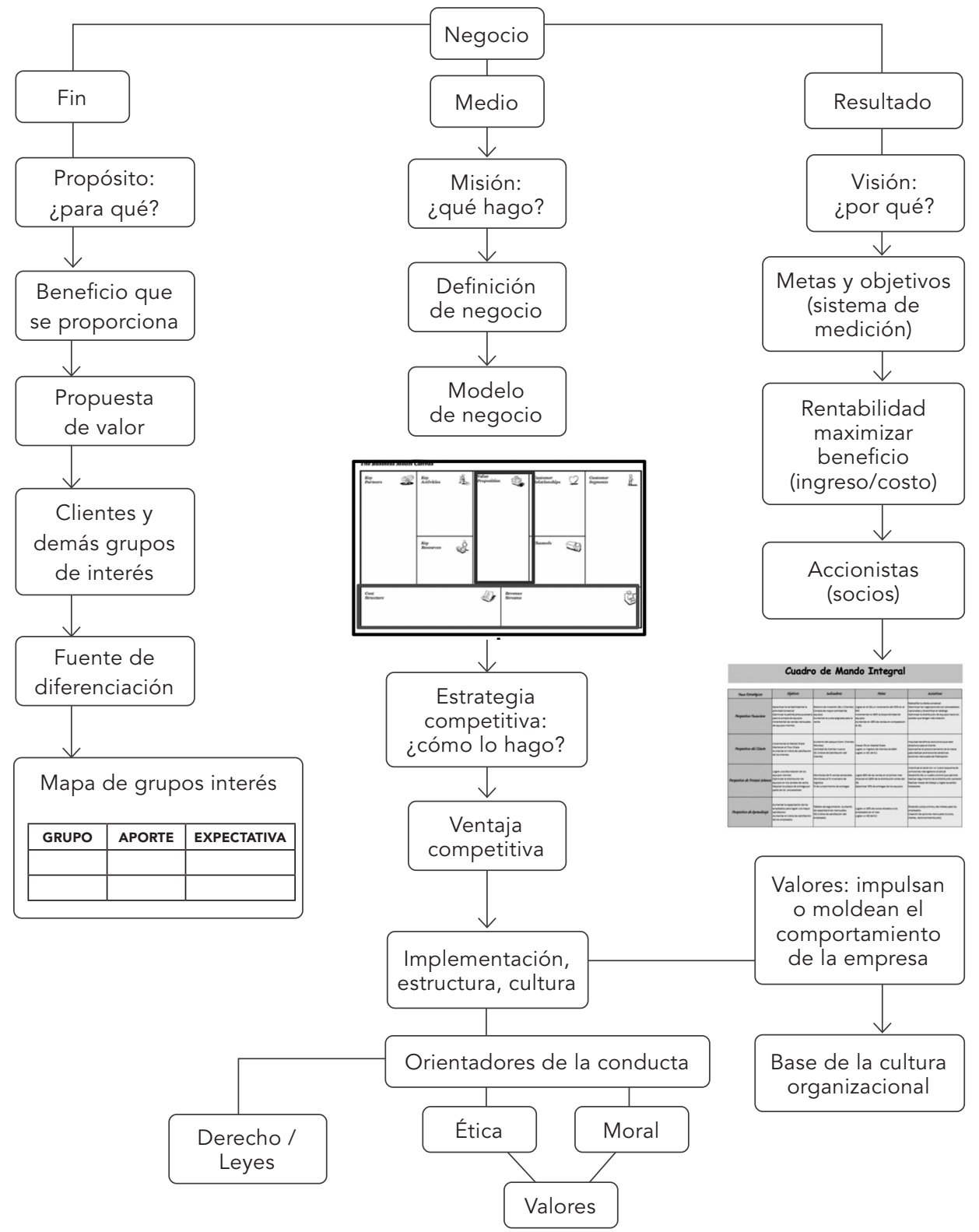

Fuente: elaboración propia, con la colaboración del ingeniero Santiago Velásquez. 
Para Cortina «[...] una organización, como una persona, no solo debe actuar con prudencia, sino también con justicia». Para lograrlo no solo debe respetar la legalidad vigente y la moral alcanzada por una sociedad, sino que también debe averiguar qué valores y derechos han de ser racionalmente respetados (Cortina, 2003: 26). Es necesaria una «[é]tica empresarial basada en el diálogo y en la confianza recíproca entre las partes implicadas en la actividad empresarial» (García-Marzá, 2004: 26). La ética es un faro porque permite identificar si una conducta, aunque esté moralmente aceptada y legalmente aprobada, puede atentar contra un valor que tiene vocación de universalidad.

La ética empresarial implica concebir la empresa como un sistema recíproco de obligaciones y expectativas, es decir, define cómo se debe comportar frente a sus grupos de interés y cómo los grupos de interés se obligan a actuar frente a esta. Ello exige valores y normas que rigen las decisiones y, además, los hábitos y conductas correspondientes. La acción moral comporta la libertad para actuar, la reciprocidad y la universalidad; esta última exige el reconocimiento y respeto de las necesidades e intereses de los demás, no considerarlos solo como recurso en el proceso de toma de decisiones (García-Marzá, 2004: 90-92), sino, como lo pensó Kant en su época, apoyado en el diálogo: «La perspectiva ética implica el abandono de la perspectiva egocéntrica y la consideración de "todos" los demás implicados y afectados no como medios para mis propios fines, sino como horizonte de sentido para mi propia acción, como fines en sí mismos» (García-Marzá, 2004: 90).

«Tener conciencia de los fines que se persiguen y habituarse a elegir y obrar en relación con ellos es la clave - como veremos- de una ética de las personas y de una ética de las organizaciones, muy especialmente de las empresas» (Cortina, 2003: 20). Debemos apoyarnos en el pilar fundamental de la ética empresarial, que es la transparencia, un valor básico para la gestión ética de la empresa (García-Marzá, 2004). La transparencia en las decisiones y acciones de la empresa fortalece su credibilidad y legitimidad frente a la sociedad.

La ética se apoya en los valores. Peter Senge se refiere a los valores y expresa que responden a la pregunta: ¿cómo queremos actuar, en coherencia con nuestra misión? Cómo quiere la empresa que sea su cotidianidad mientras persigue su visión. Los valores responden a la pregunta: ¿en qué creemos? (Senge, 1992: 283). Para Beatriz Restrepo (2014: 61) «[...] los valores se escogen, se definen, apuntando al logro del fin [...]». El carácter de universal de los valores se origina en que 
todos los ciudadanos, independientemente de sus creencias, los defienden para sí mismos; son aquellos sobre los que hay mayor nivel de acuerdo mundial. Al orientar la conducta por valores se puede lograr la finalidad de la empresa, es decir, su propósito superior, por tanto, estos contribuyen a integrar las expectativas de todos los interesados.

Se propone un enfoque que integre la racionalidad ética con la racionalidad estratégica, la ética con la economía, la ética con la estrategia, lo que exige dejar de interpretar que la empresa es una institución privada, porque requiere un trabajo cooperativo y además sus actividades afectan a otros. Por ello se espera que se centre no solo en proporcionar beneficios a los accionistas, sino también a todos los grupos de interés, lo que se traduce en confianza, legitimidad y credibilidad a la empresa, si esta logra satisfacer sus intereses legítimos. Es lo que García-Marza denomina enfoque integrativo: «El enfoque integrativo parte de la afirmación básica de que la ética y la economía no constituyen dos lógicas diferentes [...]» (García-Marza, 2004: 124-125). Se reconoce que ambas forman parte de un contexto en el que los diferentes actores sociales pueden ser afectados o pueden afectar a los demás.

\section{Metodología}

El criterio para seleccionar la literatura revisada es la contribución de los autores a la consolidación de la estrategia como campo de estudio, con alto reconocimiento por la comunidad académica. Tal es el caso de los profesores Alfred Chandler, Kenneth Andrews, Michael Porter, Henry Mintzberg y Richard Wittington, además de Donald Hambrick. También se tomaron en cuenta los contenidos de administración y dirección estratégica que han sido textos guía en el área de estrategia. Estos textos han sido objeto de revisión y selección en el Grupo de Estrategia de la Universidad EAFIT, al que pertenezco. El propósito fue acompañar la labor docente en las diferentes asignaturas que se imparten en el Área de Estrategia. Además, la labor docente durante más de 20 años en la Universidad EAFIT permitió reflexionar sobre estos textos en asignaturas como Estrategia, en el pregrado de Administración de Negocios, y Estrategia I en la Maestría en Administración de Negocios MBA, de la misma universidad.

En relación con el propósito, por ser un concepto de más reciente utilización en el ámbito organizacional y por encontrar que los autores clásicos se referían de forma 
indistinta a propósito, visión y algunas veces a misión, se adelantó una revisión en las bases de datos de la Universidad EAFIT, con el apoyo de Bertha Restrepo Restrepo (servidora de la Biblioteca «Luis Echavarría Villegas», de EAFIT), en la que se encontraron 27 artículos que hacen referencia al propósito de las organizaciones. Después de su lectura, solo se incluyeron los que tienen una relación más estrecha con el propósito como declaración que contribuye a marcar el rumbo de la empresa.

En el tema de la ética se tuvo como punto de referencia a los autores de la Escuela de Valencia, liderados por la profesora Adela Cortina. Por lo anterior, se consultaron varias de las obras de la profesora Cortina además del profesor Domingo GarcíaMarza, Jesús Conill y Juan Carlos Siurana. La fortaleza de esta escuela de éticas aplicadas reside en la prioridad que le otorga a la ética empresarial como herramienta de gestión de las organizaciones.

\section{Análisis de resultados}

\section{Modelo de coherencia estratégica: articular componentes a través de la esfera de coherencia estratégica}

Después de responder las preguntas que tienen relación con el negocio, diferenciar los conceptos correspondientes y articular la ética con la estrategia para garantizar coherencia entre las declaraciones estratégicas, y con ello integrar en la acción las expectativas de todos los interesados — con base en las fuentes bibliográficas consultadas-, se propone articular los diversos componentes a través del modelo de coherencia estratégica. A este modelo lo denomino esfera de coherencia estratégica (ver figura 2). 
Figura 2. Esfera de coherencia estratégica

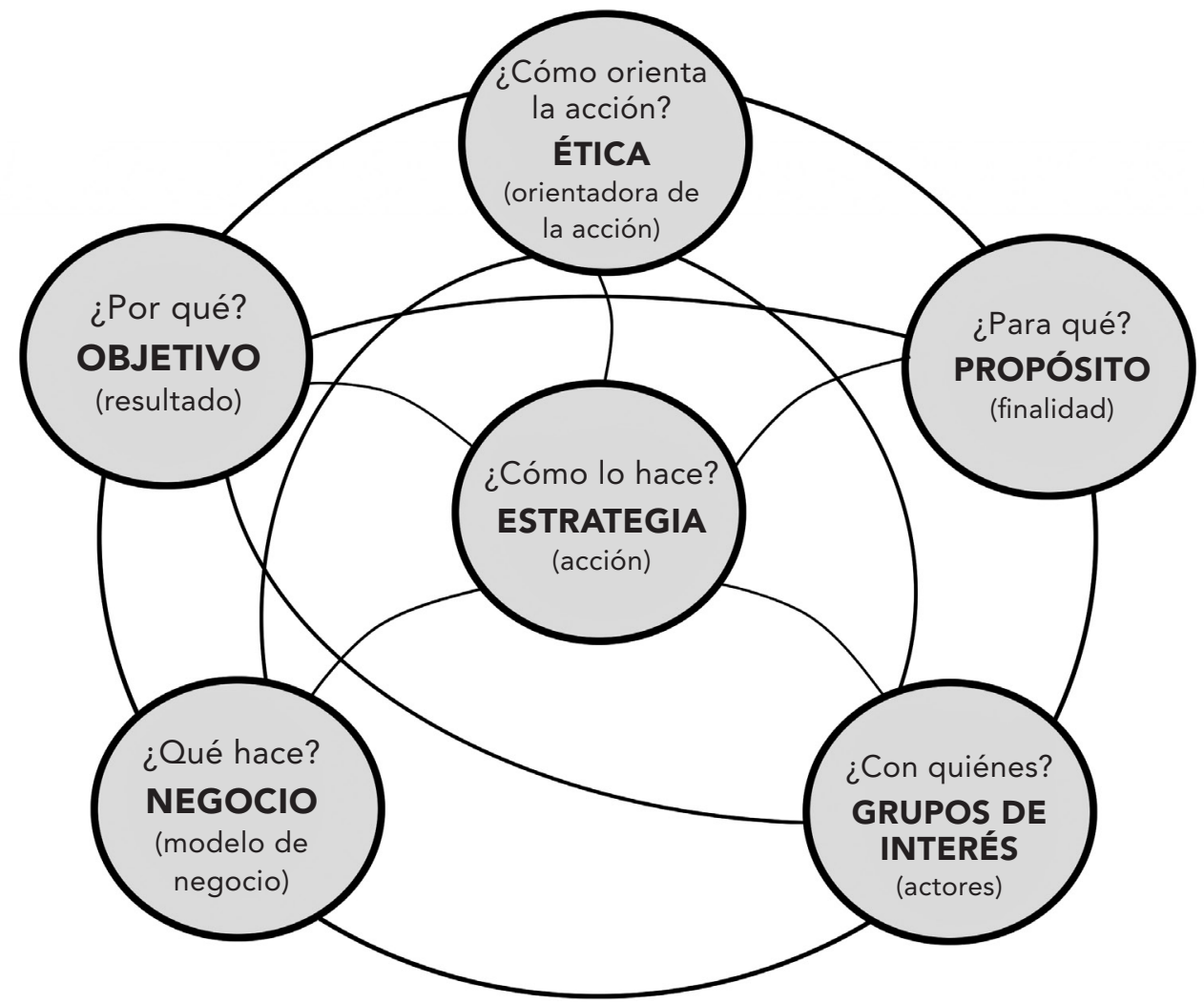

Fuente: elaboración propia con la colaboración del ingeniero Santiago Velásquez.

Los modelos, como simplificaciones de la realidad, tienen el propósito de disminuir la complejidad al seleccionar y eliminar variables. En este sentido, son de gran utilidad práctica para reflexionar sobre cuáles son las variables más relevantes que deben considerarse. La esfera de coherencia estratégica incluye seis variables a las que se ha hecho referencia en el presente artículo: 1) la ética, 2) la estrategia, 3) el propósito, 4) los objetivos, 5) el modelo de negocio y 6) los grupos de interés. La propuesta es articular estos componentes para garantizar la coherencia de la estrategia puesta en práctica por las empresas.

Todos estos componentes permiten que la estrategia sea coherente al contribuir, a través del modelo de negocio, a lograr los objetivos que se ha propuesto y, al mismo 
tiempo, proporcionar un beneficio a la sociedad o propósito, al reconocer el gran aporte que hacen para la generación de riqueza los diferentes grupos de interés; para lograrlo, orienta la acción por la ética a través de los valores.

\section{Conclusiones}

Es importante que las empresas diferencien el sentido y razón de ser de cada una de sus declaraciones estratégicas que, al ser públicas, la comprometen frente a sus diferentes grupos de interés. Para garantizar coherencia entre sus declaraciones estratégicas, pueden articularlas en torno a su modelo de negocio, de tal forma que la relación positiva entre costos e ingresos cumpla las expectativas de los accionistas y, asimismo, que la propuesta de valor refleje las expectativas de los clientes y demás grupos de interés.

Articular la ética con la estrategia contribuye a la coherencia entre las declaraciones estratégicas, y con ello puede incrementar recursos intangibles como la confianza, la credibilidad, la legitimidad, la reputación y, a su vez, estrechar relaciones con todas las partes interesadas, lo que se constituye en un recurso relacional que le asegura tener aliados.

Para garantizar coherencia estratégica se propone articular seis componentes que tienen relación con el negocio de esta forma: una estrategia orientada por la ética que, apoyada en un modelo de negocio, logra que el objetivo que le dio origen alcance el propósito que se fijó, e integre en sus acciones y decisiones las expectativas de todos los grupos de interés. 


\section{Referencias bibliográficas}

Abell, D.F. (1980). Defining the Business: The Starting Point of Strategic Planning. Nueva York: Prentice Hall.

Andrews, K. (1985). El concepto de estrategia de la empresa. Barcelona: Ediciones Orbis.

Bower, J. (1997). Oficio y arte de la gerencia. Bogotá: Grupo Editorial Norma

«Business Roundtable, la poderosa organización que ahora quiere redefinir las reglas del capitalismo» (23 de agosto de 2019). BBC News. Mundo. https://www.bbc. com/mundo/noticias-49415933

Camacho, I., Fernández, J. y Miralles, J. (2005). Ética de la empresa. Bilbao: UNIJES.

Casado, A (2008). Bioética para legos. Una introducción a la ética asistencial. España: Plaza y Valdés.

Conill, J. (2006). Horizontes de economía ética. Madrid: Editorial Tecnos.

Cortina, A. (2003). Ética de la empresa. Claves para una nueva cultura empresarial. Madrid: Editorial Trotta.

Cortina, A. (2004). Por una ética del consumo. Madrid: Editorial Taurus

Cortina, A. (2008). Ética aplicada y democracia radical. Madrid: Editorial Tecnos.

Cortina, A. (2009). «Ética de las empresas no solo responsabilidad social». Revista portuguesa de Filosofía, vol. 64 (1/4): 113-127.

Chandler, A.D. (1962). Strategy and Structure: Chapters in the History of the American Industrial Enterprise. Cambridge: MIT Press.

Schwab, Klaus (2020). Manifiesto de Davos 2020: El propósito universal de las empresas en la cuarta revolución industrial. Foro Económico Mundial. https://es. weforum.org/agenda/2019/12/manifiesto-de-davos-2020-el-proposito-universalde-las-empresas-en-la-cuarta-revolucion-industrial/

Freeman, R.E. (1984). Strategic management: a stakeholder approach. Boston: M.A. Pitman.

Friedman, Milton (13 de septiembre de 1970). «The social responsibility of business is to increase its profits». The New York Times. https://www.nytimes.com/1970/09/13/ archives/article-15-no-title.html

Friedman, M. (1997). «La responsabilidad social de los negocios es aumentar sus utilidades» (pp. 195-203). Joseph L. Bower. Oficio y arte de la gerencia. Colombia: Editorial Norma Cargraphics. 
García-Marzá, D. (2004). Ética empresarial. Del diálogo a la confianza. Madrid: Editorial Trotta.

Hambrick, D., Nag, R., Chen, M. (2007). «What is strategic management, really? Inductive derivation of a consensus definition of the field». Strategic Management Journal, vol. 28, núm. 9. https://www.scopus.com/record/display. uri?eid=2-s2.0-34547849642\&origin=inward\&txGid=0ba40ece61 f944dd 06084 b43da05b67d

Hamel, G. y Prahalad, C. K. (1990). «The core competence of the corporation». Harvard Business Review, mayo-junio, núm. 6528: 79-91.

Hamel, G. y Prahalad, C. K. (2005). «El propósito estratégico». Harvard Business Review, vol. 1. http://www.ucipfg.com/Repositorio/MAES/MAES-03/Unidad3/ Proposito_Estrategico.pdf

Hauge, A. L. y Knudsen, D. R. (2017). «Organizational purpose in management control systems: a case study on how two Norwegian organizations operationalize their purpose». Tesis de maestría en Economía, Bergen, Norwegian School of Economics. https://openaccess.nhh.no/nhh-xmlui/bitstream/handle/11250/2454022/ masterthesis.PDF? sequence=1\&isAllowed $=y$

Held, D. (2006). Modelos de democracia. Madrid: Alianza Editorial.

Hill, Ch. y Jones, G. (2011). Administración Estratégica. Un enfoque integral. México: Editorial Progreso.

Johnson, G., Scholes, K. y Whittington, R. (2005). Dirección Estratégica. Reino Unido: Pearson Prentice Hall.

Kates, A. y Galbraith, J. (2007). Designing your Organization. San Francisco: JosseyBass.

«Los jóvenes prefieren comprar los productos de las marcas que trabajan por el planeta» (23 de enero de 2020). La República, Recuperado el 27 de mayo de 2020 de: https://www.larepublica.co/responsabilidad-social/losjovenes-prefieren-comprar-los-productos-de-las-marcas-que-trabajan-por-elplaneta-2955250

Lozano, J.F. (2004). Códigos de ética para el mundo empresarial. Madrid: Editorial Trotta. 
Mintzberg, H. Quinn, J.B. y Voyer, J. (1997.) El Proceso Estratégico. Conceptos, contextos y casos. México: Prentice Hall Hispanoamérica.

Mintzberg, H., Ahlstrad, B. y Lampel, J. (2007). Safari a la estrategia: una visita guiada por la jungla de management estratégico. Buenos Aires: Granica.

Montgomery, C. (2013). El estratega. Conviértete en el líder que tu negocio necesita. Bogotá: Aguilar.

Montgomery, C. y Collins, D. (1995). «Competencia basada en recursos: estrategia de los noventa». Revista Eficiente, núm. 68: 6-21.

Naciones Unidas, ONU (2015). Objetivos de Desarrollo Sostenible. https://www. un.org/sustainabledevelopment/es/objetivos-de-desarrollo-sostenible/

Norton, D. y Kaplan, R. (2000). Cuadro de Mando Integral. Barcelona: Gestión 2000.

Osterwalder, A., y Pigneur, Y. (2011). Generación de modelos de negocio. Barcelona: Deusto.

Parker, Ceri (2020). «El Foro Económico Mundial a los 50 años de su creación: una cronología de los aspectos más destacados de Davos y más allá». World Economic Forum: 15 de enero de 2020. https://es.weforum.org/agenda/2020/01/ el-foro-economico-mundial-a-los-50-una-linea-de-tiempo-de-los-aspectos-masdestacados-de-davos-y-mas-alla-0a5878479e/

Porter, M. (1997a). «¿Qué es estrategia?». Revista Clase Empresarial 45, marzo: 81-92.

Porter, M. (1997b). «De ventaja competitiva a estrategia corporativa» (pp. 55-89). En Bower, J., Oficio y arte de la gerencia (vol. I). Bogotá: Norma.

Porter, M. (1999). Ser competitivo. Nuevas aportaciones y conclusiones. Bilbao: Editorial Deusto.

Porter, M. y Kramer M. (2006). «Empresa y sociedad. Vínculo entre ventaja competitiva y responsabilidad social corporativa». Harvard Business Review, vol. 84, núm. 12: 3-15.

Restrepo, Beatriz (2014). Reflexiones sobre educación, ética y política. Medellín: Fondo Editorial Universidad EAFIT.

Senge, P. (1992). La quinta disciplina. El arte y la práctica de la organización abierta al aprendizaje. Barcelona: Editorial Granica. 
Sinek, S. (2009). Empieza con el porqué. España: Editorial Empresa Activa.

Siurana, J. C. (2009). La sociedad ética. Indicadores para evaluar éticamente una sociedad. España: Editorial Proteus.

Wit, B. de y Meyer, R. (2010). Strategy Synthesis: Resolving Strategy Paradoxes to create competitive advantage: Resolving Strategy Paradoxes to Create Competitive Advantage (3ra ed.). Reino Unido: Cengage Learning. 


\section{Sobre la autora}

Gina Giraldo Hernández es doctora en Ética y Democracia por la Universidad de Valencia, España; Magister en Administración, MBA, por la Universidad EAFIT y titulada en Derecho y Ciencias Políticas por la Universidad de Antioquia. Profesora durante 18 años de Estrategia y, además, en los últimos tres años, del Seminario trabajo de grado. Es autora del libro Gobernanza global y justicia social.

ggirald@eafit.edu.co

https://orcid.org/0000-0002-7379-4118 